\title{
On the Drinfeld discriminant function
}

\author{
ERNST-ULRICH GEKELER \\ Fachbereich 9 Mathematik, Universität des Saarlandes, Postfach 1511 50, D-66041 Saarbrücken, \\ Germany; e-mail: gekeler@math.uni-sb.de
}

Received 9 February 1996; accepted in final form 13th February 1996

\begin{abstract}
The discriminant function $\Delta$ is a certain rigid analytic modular form defined on Drinfeld's upper half-plane $\Omega$. Its absolute value $|\Delta|$ may be considered as a function on the associated BruhatTits tree $\mathcal{T}$. We compare $\log |\Delta|$ with the conditionally convergent complex-valued Eisenstein series $E$ defined on $\mathcal{T}$ and thereby obtain results about the growth of $|\Delta|$ and of some related modular forms. We further determine to what extent roots may be extracted of $\Delta(z) / \Delta(n z)$, regarded as a holomorphic function on $\Omega$. In some cases, this enables us to calculate cuspidal divisor class groups of modular curves.
\end{abstract}

Mathematics Subject Classifications (1991): 11G18, 11G16, 11F30.

Key words: Drinfeld upper half-plane, improper Eisenstein series, modular units, cuspidal divisor class group.

\section{Notations}

We will throughout use the following notation:

$\mathbb{F}_{q} \quad=$ finite field of characteristic $p$ with $q$ elements

$A=\mathbb{F}_{q}[T]$ polynomial ring in an indeterminate $T$

$K=\mathbb{F}_{q}(T)$ rational function field

$K_{\infty}=\mathbb{F}_{q}((\pi))$ completion of $K$ at the infinite place $\left(\pi:=T^{-1}\right)$

$v_{\infty}=$ normalized valuation of $K_{\infty}$

$O_{\infty}=\mathbb{F}_{q}[[\pi]]$ integers in $K_{\infty}$

$C \quad=$ completed algebraic closure of $K_{\infty}$

|. $\mid=$ normalized absolute value on $K_{\infty}$, extended to $C$

$|.|_{i}=$ 'imaginary part': $C \rightarrow \mathbb{R},|z|_{i}=\inf _{x \in K_{\infty}}|z-x|$

$G \quad=$ group scheme GL(2)

$B \quad=$ Borel subgroup of upper triangular matrices in $\mathrm{G}$

$Z \quad=$ scalar matrices in $G$

$\mathcal{K}=G\left(O_{\infty}\right)=\operatorname{GL}\left(2, O_{\infty}\right)$

$\mathcal{I}=\left\{\left(\begin{array}{ll}a & b \\ c & d\end{array}\right) \in \mathcal{K} \mid c \equiv 0 \bmod \pi\right\}$ Iwahori subgroup

$\Gamma \quad=G(A)=\mathrm{GL}(2, A)$

$\Omega \quad=\mathbb{P}^{1}(C)-\mathbb{P}^{1}\left(K_{\infty}\right)=C-K_{\infty}$ Drinfeld upper half-plane

$\mathcal{T}=$ Bruhat - Tits tree of $\operatorname{PGL}\left(2, K_{\infty}\right)$

For any graph $\mathcal{S}$, we let $X(\mathcal{S})$ be its set of vertices, of oriented edges, respectively. For $e \in Y(\mathcal{S}), o(e), t(e) \in X(\mathcal{S})$ and $\bar{e} \in Y(\mathcal{S})$ denote its origin, terminus, and inversely oriented edge, respectively. We write 'log' for the logarithm to base $q$. 


\section{Introduction}

We let $\Gamma=\operatorname{GL}(2, A)$ be the modular group over the rational function field $K=$ $\mathbb{F}_{q}(T)$ with ring of integers $A=\mathbb{F}_{q}[T]$. The group $\Gamma$ acts on Drinfeld's upper half-plane $\Omega$, and the quotient $\Gamma \backslash \Omega$ is canonically identified with the affine line over $C=$ completed algebraic closure of $K_{\infty}=\mathbb{F}_{q}((1 / T))$.

The isomorphism is given by a $j$-invariant (the invariant of rank-two Drinfeld $A$-modules) $j=\frac{g^{q+1}}{\Delta}$, where $g$ and $\Delta$ are modular forms on $\Omega$ of respective weights $q-1$ and $q^{2}-1$. They share a number of properties with their counterparts $g_{2}, g_{3}$ and $\Delta$, respectively, in the classical modular theory: relations with Eisenstein series [13], product formulas [4], expansions around 'infinity' [7]. Further, the $C$-algebra of modular forms for $\Gamma$ is generated by $g$ and $\Delta$ (or by $g$ and the canonical $(q-1)$ th root $h$ of $\Delta$, if a 'nebentype' is admitted).

Surprisingly, not much is known so far about the behavior of the absolute values of $\Delta, g, h, j$, considered as real-valued functions on $\Omega$. Our aim in the present paper is, among others, to fill this gap.

The first main result is Theorem 2.13, where we give a formula for $|\Delta|$ as a function $\Omega \rightarrow \mathbb{R}$ that actually factors over the Bruhat-Tits tree $\mathcal{T}$ attached to $\Omega$. Corresponding expressions are given for $|j|$ and $|g|$ (Thm 2.17, Cor. 2.18). The next topic is the (related) question to what extent the functions $\Delta / \Delta_{n}$ (where $\Delta_{n}(z)=\Delta(n z), n \in A$ ) admit roots in (a) the function field of the modular curve $X_{0}(n)$ of Hecke type associated with the congruence subgroup $\Gamma_{0}(n)$ of $\Gamma$; (b) the group $\mathcal{O}(\Omega)^{*}$ of invertible holomorphic functions on $\Omega$. The answer is given in Theorem 3.16 and its corollaries. We further determine the character $\omega_{n}$ of $\Gamma_{0}(n)$ through which $\Gamma_{0}(n)$ acts on the 'maximal root' $D_{n}$ of $\Delta / \Delta_{n}$ (Thm $3.20+$ Cor. 3.21). These questions are connected with the structure of the cuspidal divisor class group of $X_{0}(n)$, as is demonstrated in the concluding Examples 3.23 and 3.25 .

Our main technical tools are the 'logarithmic derivative' mapping $r: \mathcal{O}(\Omega)^{*} \rightarrow$ $\underline{H}(\mathcal{T}, \mathbb{Z})$ (see (1.10)) and Fourier analysis on the tree $\Gamma_{\infty} \backslash \mathcal{T}$, where $\Gamma_{\infty}=$ $\left\{\left(\begin{array}{ll}a & b \\ 0 & d\end{array}\right) \in \Gamma\right\}$. We compare $r(\Delta)$ with the improper Eisenstein series $E$ on $\mathcal{T}$ defined by complex analytic means [8]. Since $r(\Delta)$ and $E$ agree up to a constant (Cor. (2.8)), we can derive properties of $\Delta$ from the properties of $E$ shown in [8]. This way there results e.g. an upper bound for $\max \left\{i \mid \Delta / \Delta_{n}\right.$ has an $i$ th root $\}$. We then verify it is sharp by constructing such a root through modular forms.

\section{Drinfeld modular forms, logarithmic derivatives, Fourier coefficients}

A $C$-valued function $f$ on the 'upper half-plane' $\Omega=C-K_{\infty}$ is a modular form of weight $k \in \mathbb{N}_{0}$ and type $m \in \mathbb{Z} /(q-1)$ for $\Gamma=\mathrm{GL}(2, A)$ if 
(i) $\quad f\left(\frac{a z+b}{c z+d}\right)=(c z+d)^{k}(\operatorname{det} \gamma)^{-m} f(z)$,

$$
=\gamma\left(\begin{array}{l}
a b \\
c d
\end{array}\right) \in \Gamma, z \in \Omega
$$

(ii) $f$ is holomorphic (in the rigid analytic sense);

(iii) $f$ is holomorphic at infinity.

The description of the analytic structure on $\Omega$ is given e.g. in $[1,2,9,13]$; the meaning of condition (iii) is explained in [7] 5.7. Similarly, we define modular forms for subgroups $\Gamma^{\prime} \subset \Gamma$ of finite index. Besides the Eisenstein series

$$
E^{(k)}(z)=\sum_{a, b \in A}^{\prime}(a z+b)^{-k} \quad(0<k \equiv 0 \bmod q-1),
$$

which are modular of weight $k$ and type 0 [13], there are three distinguished modular forms $g, h, \Delta$ which, among others, enjoy the following properties (see [7] for a systematic presentation; here we use the normalization $g=g_{\text {old }}, \Delta=\Delta_{\text {old }}$ of loc. cit. p. 683, which involves a slight change of constants in some formulas).

Let $M_{k, m}$ be the $C$-vector space of forms of weight $k$ and type $m$. Then

$$
\begin{aligned}
& g=\left(T^{q}-T\right) E^{(q-1)} \in M_{q-1,0} \\
& \Delta=\left(T^{q^{2}}-T\right) E^{\left(q^{2}-1\right)}+\left(T^{q^{2}}-T^{q}\right) E^{(q-1)^{q+1}} \in M_{q^{2}-1,0} \\
& \text { vanishes nowhere on } \Omega \\
& h=g^{\prime}-\frac{\Delta^{\prime}}{\Delta} g \in M_{q+1,1} \quad\left(f^{\prime}=\bar{\pi} \frac{\mathrm{d} f}{\mathrm{~d} z}\right) \\
& h^{q-1}=-\Delta \\
& \bigoplus M_{k, 0}=C[g, \Delta] \quad(\text { David Goss [14]) } \\
& k \geqslant 0 \\
& k \geqslant 0, m \in \mathbb{Z} /(q-1)
\end{aligned}
$$

Here $\bar{\pi} \in C$ is some constant analogous with $2 \pi i$, with logarithmic absolute value $\log |\bar{\pi}|=\frac{q}{q-1}$. Note that $f \mapsto f^{\prime}$ is $\bar{\pi}^{2}$ times the operator $\theta$ of [7], which compensates the different normalizations of $g, h$, and $\Delta$. These forms naturally appear as the coefficients of Drinfeld modules. Whereas $g$ is similar to the coefficient forms $g_{2}, g_{3}$ in the theory of elliptic curves, the Drinfeld discriminant $\Delta$ shares many of the properties of the classical discriminant $\Delta(z)=(2 \pi i)^{12} \Pi\left(1-\mathrm{e}^{2 \pi i n z}\right)^{24}$.

Next, let $\mathcal{T}$ be the Bruhat-Tits tree of $\operatorname{PGL}\left(2, K_{\infty}\right)$. It is a $(q+1)$-regular tree with

$$
\begin{array}{ll}
X(\mathcal{T})=G\left(K_{\infty}\right) / \mathcal{K} \cdot \mathcal{Z}\left(\mathcal{K}_{\infty}\right) & \text { (vertices) } \\
Y(\mathcal{T})=G\left(K_{\infty}\right) / \mathcal{I} \cdot \mathcal{Z}\left(\mathcal{K}_{\infty}\right) & \text { (orientied edges) }
\end{array}
$$


where the canonical map from $Y(\mathcal{T})$ to $X(\mathcal{T})$ associates with each edge $e$ its origin $o(e)$. It is easily verified that

$$
S_{X}:=\left\{\left(\begin{array}{cc}
\pi^{k} & u \\
0 & 1
\end{array}\right) \mid \begin{array}{l}
k \in \mathbb{Z}, u \in K_{\infty}, \\
u \bmod \pi^{k} O_{\infty}
\end{array}\right\}
$$

is a set of representatives for $X(\mathcal{T})$. We let $v(k, u)$ be the vertex corresponding to $\left(\begin{array}{cc}\pi^{k} & u \\ 0 & 1\end{array}\right)$. By an end of $\mathcal{T}$, we understand the equivalence class of an infinite path without backtracking, where two paths that differ in a finite number of edges are identified. The set $\partial \mathcal{T}$ of ends of $\mathcal{T}$ is in $1-1$ correspondence with $\mathbb{P}^{1}\left(K_{\infty}\right)=$ space of lines in $V=K_{\infty}^{2}$. We normalize the bijection such that the end $(v(k, 0)$, $v(k-1,0), \ldots)$ corresponds to $\infty$. It defines an orientation on $\mathcal{T}$, i.e., a decomposition $Y(\mathcal{T})=Y^{+}(\mathcal{T}) \dot{\cup} Y^{-}(\mathcal{T})$ with $\overline{Y^{+}(\mathcal{T})}=Y^{-}(\mathcal{T})$. Namely, $e \in Y(\mathcal{T})$ is positive $\left(\Leftrightarrow e \in Y^{+}(\mathcal{T}) \Leftrightarrow \operatorname{sgn}(e)=+1\right)$ if it points to the end $\infty$, end negative ( $\Leftrightarrow e \in Y^{-}(\mathcal{T}) \Leftrightarrow \operatorname{sgn}(e)=-1$ ) otherwise. We thus get a section

$$
\begin{aligned}
X(\mathcal{T}) & \cong Y^{+}(\mathcal{T}) \hookrightarrow Y(\mathcal{T}) \\
v & \longmapsto e \text { s.t. } o(e)=v, \operatorname{sgn}(e)=+1
\end{aligned}
$$

for the 'origin' map from $Y(\mathcal{T})$ to $X(\mathcal{T})$. Since the reflection e $\mapsto \bar{e}$ on $Y(\mathcal{T})$ group-theoretically is given by

$$
\text { class of } g \in G\left(K_{\infty}\right) \mapsto \text { class of } g\left(\begin{array}{cc}
0 & 1 \\
\pi & 0
\end{array}\right),
$$

each $e \in Y(\mathcal{T})$ is uniquely represented by

$$
\begin{aligned}
& \text { either }\left(\begin{array}{cc}
\pi^{k} & u \\
0 & 1
\end{array}\right) \quad(\text { if } \operatorname{sgn}(e)=+1, \\
& \text { in this case we put } e=: e(k, u) \text { ) } \\
& \text { or } \quad\left(\begin{array}{ll}
\pi^{k} & u \\
0 & 1
\end{array}\right)\left(\begin{array}{ll}
0 & 1 \\
\pi & 0
\end{array}\right) \quad(\text { if } \operatorname{sgn}(e)=-1)
\end{aligned}
$$

with $\left(\begin{array}{cc}\pi^{k} & u \\ 0 & 1\end{array}\right) \in S_{X}$. Now each such element of $S_{X}$ with $u \notin \pi^{k} O_{\infty}$ may be written as

$$
\left(\begin{array}{cc}
\pi^{a+t}, & \pi^{t} v \\
0, & 1
\end{array}\right) \quad \text { with } t \in \mathbb{Z}, a \in \mathbb{N} \text { uniquely determined, }
$$

and $v \in O_{\infty}^{*}$ uniquely determined modulo $\pi^{a} O_{\infty}$. We define the functions $\kappa, \tau, \alpha$ on $Y(\mathcal{T})$ by

$$
\begin{array}{ll}
\kappa(e)=k, \tau(e)=t, \alpha(e)=a, & e \text { or } \bar{e} \text { equal to } e(k, u), u \notin \pi^{k} O_{\infty}, \\
\kappa(e)=\tau(e)=k, \alpha(e)=0, & e \text { or } \bar{e} \text { equal to } e(k, 0) .
\end{array}
$$


By definition, $\kappa, \tau$ and $\alpha$ are invariant under $e \mapsto \bar{e}$, and $\kappa$ is invariant under the action of the stabilizer

$$
\Gamma_{\infty}=\Gamma \cap B
$$

of the end $\infty$ in $\Gamma$. The intuitive meaning is as follows: Let $A(0, \infty)$ be the principal axis of $\mathcal{T}$, i.e., the path $(\ldots, e(k+1,0), e(k, 0), e(k-1,0), \ldots)$ from the end 0 to the end $\infty$ of $\mathcal{T}$. Then $\alpha(e)$ is the distance from $e$ to $A(0, \infty), \tau(e)$ describes the vertex next to $e$ on $A(0, \infty)$, and $\kappa$ decreases by one on each step towards $\infty$.

We put $\underline{H}(\mathcal{T}, \mathbb{Z})$ for the group (and right $G\left(K_{\infty}\right)$-module) of maps $\varphi: Y(\mathcal{T}) \rightarrow \mathbb{Z}$ that satisfy

(i) $\varphi(e)+\varphi(\bar{e})=0, e \in Y(\mathcal{T}) \quad(\varphi$ alternating $)$

(ii) $\sum_{\substack{e \in Y(\mathcal{T}) \\ o(e)=v}} \varphi(e)=0, \quad v \in X(\mathcal{T})$ ( $\varphi$ harmonic).

$\underline{H}(\mathcal{T}, \mathbb{Z})$ is called the module of integral-valued harmonic cochains or currents. Both $\Omega$ and $\mathcal{T}$ are analogues of the complex upper half-plane; they are related by a $G\left(K_{\infty}\right)$-equivariant map $\lambda: \Omega \rightarrow \mathcal{T}(\mathbb{R})$ (= points of the realization of $\mathbb{R}$ ) that we will briefly describe. Recall [11] that $\mathcal{T}(\mathbb{R})$ may be canonically identified with the set of equivalence classes of norms on the two-dimensional $K_{\infty}$-vector space $K_{\infty}^{2}$. Then $\lambda(z)$ corresponds to the norm $\nu_{z}$, where $\nu_{z}((u, v)):=|u z+v|$. The map $\lambda$ is onto the rational points $\mathcal{T}(\mathbb{Q})$ of $\mathcal{T}$. We have in $\Omega \subset \mathbb{P}^{1}(C)$ :

$$
\begin{aligned}
& \lambda^{-1}(\text { vertex }) \cong \mathbb{P}^{1}(C)-(q+1) \text { disjoint balls, } \\
& \lambda^{-1}\left(\begin{array}{c}
\text { edge minus } \\
\text { end points }
\end{array}\right) \cong \mathbb{P}^{1}(C)-\text { two disjoint balls. }
\end{aligned}
$$

For example,

$$
\begin{aligned}
\lambda^{-1}(v(0,0)) & =\{z \in C|| z|\leqslant 1,| z-c \mid \geqslant 1, \\
& \left.\forall c \in \mathbb{F}_{q}\right\} \\
= & \left\{\left.z \in C|| z|=| z\right|_{i}=1\right\} \quad \text { and } \\
\lambda^{-1}(e(0,0)-\text { end points })= & \{z \in C|1<| z \mid<q\} .
\end{aligned}
$$

The relationship between the functions $||,.|.|{ }_{i}$ on $C$ and the functions $\kappa, \tau, \alpha$ on $\mathcal{T}$ is as follows.

1.8 LEMMA. Let $z \in \Omega$ be such that $\lambda(z)=v \in X(\mathcal{T})$, and let $e$ be the unique positive edge with $o(e)=v$. Then

$$
\log |z|_{i}=-\kappa(e) \quad \text { and } \quad \log |z|=-\tau(e) .
$$


Proof. Straightforward from (1.7), the $G\left(K_{\infty}\right)$-equivariance of $\lambda$, and the formula

$$
|\gamma z|_{i}=|c z+d|^{-2}|\operatorname{det} \gamma||z|_{i}
$$

for $\gamma=\left(\begin{array}{l}a b \\ c d\end{array}\right) \in G\left(K_{\infty}\right)$.

1.9 COROLLARY. For $z \in \Omega$, the conditions (i) $|z|=|z|_{i}$ and (ii) $\lambda(z) \in A(0, \infty)$ are equivalent.

Proof. Without restriction, $\lambda(z) \in X(\mathcal{T})$ since both $\log |z|$ and $\log |z|_{i}$ factor through $\lambda$ and are linear on edges of $\mathcal{T}$. Then the assertion is clear from the lemma and (1.5).

The following construction, due to Marius van der Put, is fundamental for the study of modular forms. Let $\mathcal{O}=\mathcal{O}_{\Omega}$ be the structure sheaf of the analytic space $\Omega$ and $\mathcal{O}(\Omega)^{*}$ the units of its global sections. Then there is a canonical short exact sequence of $G\left(K_{\infty}\right)$-modules (trivial action on $C^{*}$ ):

$$
0 \rightarrow C^{*} \rightarrow \mathcal{O}(\Omega)^{*} \stackrel{\tau}{\rightarrow} \underline{H}(\mathcal{T}, \mathbb{Z}) \rightarrow 0 .
$$

It is related with the logarithmic derivative through the commutative diagram

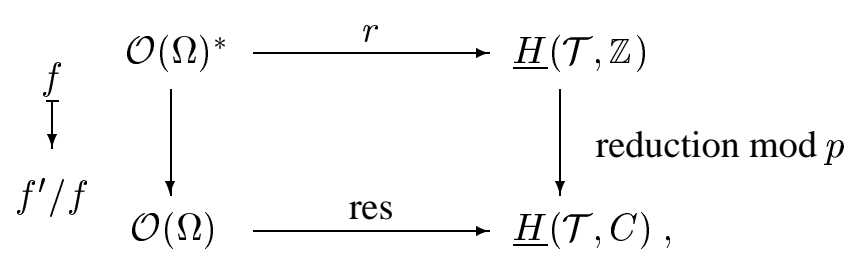

where the lower horizontal map is res: $g(z) \mapsto \operatorname{res} g(z) \mathrm{d} z,(\operatorname{res} \omega)(e)=$ residue of the differential form $\omega$ in the oriented annulus $\lambda^{-1}(e)$. The definition of $r$ is as follows:

$$
r(f)(e)=\log \frac{\|f\|_{\lambda^{-1}(t(e))}}{\|f\|_{\lambda^{-1}(o(e))}},
$$

where $\|f\|_{\lambda^{-1}(v)}$ denotes the spectral norm of $f \in \mathcal{O}(\Omega)^{*}$ on $\lambda^{-1}(v)$, i.e., $\sup \left\{|f(z)| \mid z \in \lambda^{-1}(v)\right\}=|f(z)|\left(z \in \lambda^{-1}(v)\right)$ since $f$ is invertible. The fact that $r$ is well-defined (i.e., takes its values in $\underline{H}(\mathcal{T}, \mathbb{Z})$ ) and has the stated properties is proved in [2] and [10]. In particular, we have for $f \in \mathcal{O}(\Omega)^{*}$, $v_{1}, v_{2} \in X(\mathcal{T}), z_{1}, z_{2} \in \Omega$ with $\lambda\left(z_{i}\right)=v_{i}$ :

$$
\log \left|\frac{f\left(z_{2}\right)}{f\left(z_{1}\right)}\right|=\int_{v_{1}}^{v_{2}} r(f)(e) \mathrm{d} e
$$


(Recall that ' $\log$ ' $={ }^{\prime} \log _{q}$ '. The integral is the sum of $r(f)$ along the unique path from $v_{1}$ to $v_{2}$.) In view of (1.10) to (1.13), we like to view $r$ as a substitute for the logarithmic derivative map $f \mapsto f^{\prime} / f$ in the classical theory. The next result calculates $r(f)$ for the most elementary functions $f \in \mathcal{O}(\Omega)^{*}$.

1.14 PROPOSITION. Let $a \neq b \in \mathbb{P}^{1}\left(K_{\infty}\right)$ and $f_{a, b}$ be a rational function on $\mathbb{P}^{1}(C)$ with a simple zero at $a$, a simple pole at $b$ and no further zeroes and poles. Let further $A(a, b)$ be the unique path in $\mathcal{T}$ from the end a to the end $b$, and define $\varphi_{a, b} \in \underline{H}(\mathcal{T}, \mathbb{Z})$ by

$$
\varphi_{a, b}(e)=\left\{\begin{array}{cl}
1 & e \text { on } A(a, b) \\
-1 & \bar{e} \text { on } A(a, b) \\
1 & \text { otherwise }
\end{array}\right.
$$

Then $r\left(f_{a, b}\right)=\varphi_{a, b}$.

Proof. In view of the $G\left(K_{\infty}\right)$-equivariance of $r$, it suffices to consider the case $(a, b)=(0, \infty)$, i.e., $f(z)=z$ the identity function. Let $z \in \Omega$ be such that $\lambda(z)=v=o(e) \in X(\mathcal{T})$ with some $e \in Y^{+}(\mathcal{T}), e^{\prime} \in Y^{+}(\mathcal{T})$ the first edge on the path from $v$ to $\infty$ lying on the axis $A(0, \infty), v^{\prime}=o\left(e^{\prime}\right)$. Suppose that $e \neq e^{\prime}$. The picture looks:

$$
\begin{gathered}
e=e(k, *) \\
e^{\prime}=e(t, 0) \\
t=\tau(e) \\
k-t=\alpha(e)=\operatorname{distance}\left(v, v^{\prime}\right)
\end{gathered}
$$

Next, we associate Fourier coefficients to $\Gamma_{\infty}$-invariant elements $\varphi$ of $\underline{H}(\mathcal{T}, \mathbb{C})$. In view of (1.4) and (1.6)(i), each $\varphi \in \underline{H}(\mathcal{T}, \mathbb{C})$ is uniquely determined by its restriction to $Y^{+}(\mathcal{T})=B\left(K_{\infty}\right) /\left(I \cap B\left(K_{\infty}\right)\right) Z\left(K_{\infty}\right)$. We may thus regard $\varphi \in$ $\underline{H}(\mathcal{T}, \mathbb{C})^{\Gamma_{\infty}}$ as a function on $Y^{+}\left(\Gamma_{\infty} \backslash \mathcal{T}\right)=\Gamma_{\infty} \backslash Y^{+}(\mathcal{T})$, the positive edges of the quotient $\Gamma_{\infty} \backslash \mathcal{T}$ by $\Gamma_{\infty}=\Gamma \cap B$, and apply the machinery of Fourier analysis. The following is an adaption of [19] Ch. III to our situation. Details are carried out in [8], Sections 2 and 3. 
Let $\operatorname{Div}(K)$ be the multiplicative group of divisors on $K$ and $\operatorname{Div}^{+}(K) \hookrightarrow$ $\operatorname{Div}(K)$ the monoid of positive divisors. Each $\mathfrak{m} \in \operatorname{Div}(K)$ may uniquely be written as a power $\infty^{k}$ of the infinite prime $\infty$ times a finite divisor $\mathfrak{m}_{f}$ (i.e., $\infty \notin$ $\left.\operatorname{supp}\left(\mathfrak{m}_{f}\right)\right)$. We identify finite positive divisors with ideals of $A=\mathbb{F}_{q}[T]$. The norm $|\mathfrak{m}|$ of $\mathfrak{m} \in \operatorname{Div}(K)$ is $q^{\operatorname{deg} \mathfrak{m}}$. The principal $\operatorname{divisor} \operatorname{div}(m)$ of $m \in K^{*}$ is always understood with its infinity part, so that its degree is zero. For $u=\Sigma u_{i} \pi^{i} \in K_{\infty}$, put $\nu(u)=q-1$ if $u_{1}=0$ and $\nu(u)=-1$ otherwise. Then we define for each $\varphi \in \underline{H}(\mathcal{T}, \mathbb{C})^{\Gamma_{\infty}}$ two functions

$$
\begin{array}{ll}
c_{0}(\varphi, \cdot): K_{\infty}^{*} & \rightarrow \mathbb{C} \text { (the constant Fourier coefficient of } \varphi \text { ) } \\
c(\varphi, \cdot): \operatorname{Div}^{+}(K) \rightarrow \mathbb{C} \text { (the nonconstant Fourier coefficient of } \varphi \text { ) }
\end{array}
$$

by

$$
\begin{aligned}
c_{0}(\varphi, x) & =q^{1-k} \sum_{u \in(\pi) /\left(\pi^{k}\right)} \varphi(e(k, u)) \quad k:=v_{\infty}(x) \geqslant 1 \\
& =\varphi(e(k, 0)) \quad k \leqslant 1 \\
c(\varphi, \mathfrak{m}) & =q^{-1-l}\left[\sum_{\substack{0 \neq u \in(\pi) /\left(\pi^{2+l}\right) \\
\text { monic }}} \varphi(e(2+l, u)) \nu(-m u)+\varphi(e(2+l, 0))\right]
\end{aligned}
$$

if $\mathfrak{m}=\operatorname{div}(m) \cdot \infty^{l}$ with some $m \in A$.

The $\Gamma_{\infty}$-invariance of $\varphi$ implies that the summands appearing on the right hand sides only depend on the respective residue classes of $u \in(\pi)=\pi O_{\infty}$. Some $u \in K_{\infty}^{*}$ is monic if its lowest order coefficient in $\pi$ is one. Again from the $\Gamma_{\infty^{-}}$ invariance, we could replace the sum over the nonzero monics in $(\pi) /\left(\pi^{2+l}\right)$ by the sum over the nonzero $u \in\left[(\pi) /\left(\pi^{2+l}\right)\right] / \mathbb{F}_{q}^{*}$. The Fourier coefficients satisfy

$$
\begin{aligned}
& \text { (i) } c_{0}(\varphi, x)=q^{-v_{\infty}(x)} c_{0}(\varphi, 1)=q^{-v_{\infty}(x)} \varphi(e(0,0)) \text {; } \\
& \text { (ii) } c\left(\varphi, \mathfrak{m}_{f} \cdot \infty^{k}\right)=q^{-k} c\left(\varphi, \mathfrak{m}_{f}\right) \quad\left(k \in \mathbb{N}_{0}\right) \text {; } \\
& \text { (iii) } \varphi(e(k, u))=c_{0}\left(\varphi, \pi^{k}\right) \\
& +\sum_{\substack{m \in A \text { monic } \\
\text { deg } \\
m \leqslant k-2}} c\left(\varphi, \operatorname{div}(m) \cdot \infty^{k-2}\right) \nu(m u) .
\end{aligned}
$$

Properties (i) and (ii) reflect the harmonicity of $\varphi$ as a function on $Y^{+}(\mathcal{T})$, and (iii) is the inversion formula. In (iii) and similar expressions, $c(\varphi, \mathfrak{m})=0$ if $\mathfrak{m}$ fails to be positive. Conversely, given functions $c_{0}$ and $c$ that satisfy (i) and (ii), the function $\varphi$ defined by (iii) lies in $\underline{H}(\mathcal{T}, \mathbb{C})^{\Gamma \infty}$. 
We finally introduce Hecke operators. For a function $\varphi$ on $Y^{+}(\mathcal{T})=$ $B\left(K_{\infty}\right) /\left(\mathcal{I} \cap B\left(K_{\infty}\right)\right) Z\left(K_{\infty}\right)$ and a positive finite divisor $\mathfrak{m}$, we put

$$
T_{\mathrm{m}} \varphi(x)=\sum \varphi\left(\left(\begin{array}{ll}
a & b \\
0 & d
\end{array}\right) x\right) \quad\left(x \in B\left(K_{\infty}\right)\right),
$$

where the sum is over $a, b, d \in A$ such that $a, d$ are monic, $(a d)=\mathfrak{m}$ and $\operatorname{deg} b<$ $\operatorname{deg} d$. Then $T_{\mathfrak{m}} \varphi$ is again a function on $Y^{+}(\mathcal{T})$ (i.e., right $\left(\mathcal{I} \cap B\left(K_{\infty}\right)\right) Z\left(K_{\infty}\right)$ invariant as a function on $\left.B\left(K_{\infty}\right)\right)$ and even in $\underline{H}(\mathcal{T}, \mathbb{C})^{\Gamma \infty}$ if $\varphi$ is. The $T_{\mathfrak{m}}$ have the usual properties, which may be looked up in [19] Ch. VI. We just point out that we can read off from its Fourier coefficients that $\varphi \in \underline{H}(\mathcal{T}, \mathbb{C})^{\Gamma_{\infty}}$ is an eigenform (loc. cit. p. 44).

\section{The logarithmic derivative of the discriminant}

We now calculate the current $r(\Delta)$ and derive some consequences. The functional equation

$$
\Delta(\gamma z)=(c z+d)^{q^{2}-1} \Delta(z) \quad\left(\gamma=\left(\begin{array}{ll}
a & b \\
c & d
\end{array}\right) \in \Gamma\right)
$$

translates to

$$
r(\Delta)(\gamma e)=\left(q^{2}-1\right) \varphi(e)+r(\Delta)(e),
$$

where by (1.13), $\varphi \in \underline{H}(\mathcal{T}, \mathbb{Z})$ equals $\varphi_{-d / c, \infty}$ if $c \neq 0$ and $\varphi=0$ otherwise. Now

$$
\varphi(e) \neq 0 \Leftrightarrow c \neq 0 \quad \text { and } \quad\left(\begin{array}{cc}
c & d \\
0 & 1
\end{array}\right)(e) \in A(0, \infty),
$$

in which case $\varphi(e)=\operatorname{sgn}(e)$. We therefore define

$$
\begin{array}{rlrl}
S(\gamma, e):=\operatorname{sgn}(e), & \text { if } \gamma=\left(\begin{array}{ll}
a & b \\
c & d
\end{array}\right) \text { with } c \neq 0 \\
& & \text { and }\left(\begin{array}{ll}
c & d \\
0 & 1
\end{array}\right)(e) \in A(0, \infty) \\
=0 & & \text { otherwise. }
\end{array}
$$

2.2 LEMMA. (i) $S(\gamma, e)=0 \Leftrightarrow \operatorname{sgn}(e)=\operatorname{sgn}(\gamma e)$.

(ii) $S(\gamma \delta, e)=S(\gamma, \delta e)+S(\delta, e), \gamma, \delta \in \Gamma$. 
Proof. (i) [8] (4.5) + (4.6). (ii) Straightforward calculation.

Using $S(\gamma, e)$, we may thus express the behavior of $r(\Delta)$ under $\Gamma$ by the functional equation

$$
r(\Delta)(\gamma e)=\left(q^{2}-1\right) S(\gamma, e)+r(\Delta)(e) .
$$

On the other hand, it is clear that (2.3) characterizes $r(\Delta)$ : If $\varphi$ is another element of $\underline{H}(\mathcal{T}, \mathbb{Z})$ subject to the same transformation rule, the difference $r(\Delta)-\varphi$ is $\Gamma$-invariant. But it is well-known that the quotient $\Gamma \backslash \mathcal{T}$ is a half-line

$$
\Gamma \backslash \mathcal{T}=\bullet---\bullet---\bullet---\bullet \cdots
$$

represented by the vertices $v(k, 0), k \leqslant 0$ (e.g. [17] p. 111), and therefore $\underline{H}(\mathcal{T}, \mathbb{Z})^{\Gamma}=0$ and $r(\Delta)=\varphi$.

Let now $E \in \underline{H}(\mathcal{T}, \mathbb{C})^{\Gamma}$ be the current defined through its Fourier coefficients $c_{0}()=.c_{0}(E,),. c()=.c(E,$.$) :$

(i) $c_{0}\left(\pi^{k}\right)=-\frac{q^{2}}{q^{2}-1} q^{-k}$;

(ii) $c_{0}$ is Eulerian ([19] p. 10) at $\infty$ with Euler factor $\left(1-q^{-1} X\right)^{-1}$;

(iii) $c_{0}$ is Eulerian at finite places $\mathfrak{p}$ of $K$ with Euler factor $\left(1-\left(1+|\mathfrak{p}|^{-1}\right) X+|\mathfrak{p}|^{-1} X^{2}\right)^{-1}$

(iv) $c((1))=1$.

As can be read off from the Fourier coefficients, $E$ is an eigenform for the Hecke operator $T_{\mathfrak{p}}$ with eigenvalue $\epsilon_{\mathfrak{p}}=1+|\mathfrak{p}|$. The next result is proved in [8] Theorem 6.1, Corollary 6.2, Proposition 5.8:

2.6 THEOREM. (i) For each $\gamma \in \Gamma$, E satisfies the functional equation

$$
E(\gamma e)=\frac{q}{q-1} S(\gamma, e)+E(e)
$$

(ii)

$$
\begin{aligned}
E(e(k, 0)) & =-\frac{q^{2}}{q^{2}-1} q^{-k} & & k \leqslant 1 \\
& =\frac{q^{k+1}-q^{2}-q}{q^{2}-1} & & k \geqslant 1 .
\end{aligned}
$$

(iii) The set of values (up to sign) of $E$ on $Y(\mathcal{T})$ is contained in the set of values on $A(0, \infty)$ described by (ii). In particular, its values are rational with bounded 
denominator $\left(q^{2}-1\right)$.

2.7 Remark. E may also be represented, up to a scalar factor, as an improper (= only conditionally convergent) Eisenstein series $\sum_{\gamma \in \Gamma_{\infty} \backslash \Gamma} \varphi(\gamma e)$, where $\varphi(e)=$ $\operatorname{sgn}(e) q^{-\kappa(e)}$, and where the summation has to be taken in a fixed order (loc. cit.).

Comparing the constants in the respective functional equations, we have the immediate corollaries.

\subsection{COROLLARY.}

$$
r(\Delta)=\frac{\left(q^{2}-1\right)(q-1)}{q} E
$$

and

$$
r(h)=\frac{q^{2}-1}{q} E
$$

\subsection{COROLLARY.}

$$
\begin{aligned}
r(\Delta)(e(k, 0)) & =-q^{1-k}(q-1) & & k \leqslant 1 \\
& =\left(q^{k}-q-1\right)(q-1) & & k \geqslant 1
\end{aligned}
$$

and this gives (up to sign) all the values of $r(\Delta)$ on $Y(\mathcal{T})$.

2.10 COROLLARY. Let $r$ be the largest number such that there exists an $r$ th root of $\Delta$ in $\mathcal{O}(\Omega)^{*}$. Then $r=q-1$.

Proof. $\operatorname{gcd}\{$ values of $r(\Delta)\}=q-1$.

2.11 COROLLARY. Let $\mathfrak{p}=(f) \subset A$ be a prime, $f$ monic. The function $h$ (and hence $\left.\Delta=-h^{q-1}\right)$ satisfies the functional equation

$$
h(f z) \prod_{\substack{b \in A \\ \operatorname{deg} b<\operatorname{deg} \mathfrak{p}}} h\left(\frac{z+b}{f}\right)=h^{|\mathfrak{p}|+1}(z) .
$$

Proof. Let $\widetilde{h}$ be the left hand side. Then $r(\widetilde{h})=T_{\mathfrak{p}} r(h)=(|\mathfrak{p}|+1) r(h)$, where the first equation is immediate from the definition of Hecke operators, and the second one results from (2.5) and (2.8). Hence $\widetilde{h}(z)=$ const. $h^{|\mathfrak{p}|+1}(z)$, and the constant is determined to 1 using the expansion of $h$ around the cusp $\infty$ ([7] Theorem 9.1).

2.12 Remark. The above formula may be written more suggestively as

$$
\frac{h(z)}{h(f z)}=\prod_{\substack{b \in A \\ \operatorname{deg} b<\operatorname{deg} \mathfrak{p}}} \frac{h\left(\frac{z+b}{f}\right)}{h(z)},
$$


i.e., as a distribution relation. It is then similar to the distribution relations satisfied by the classical discriminant $\Delta$ and related functions (see $[15,16]$ ). Clearly, we can write down relations analogous to (2.11) for not necessarily prime ideals $\mathfrak{m} \subset A$, exploiting the fact that $r(h)$ is an eigenform for $T_{\mathfrak{m}}$.

2.13 THEOREM. Let $z_{k} \in \Omega$ be such that $\lambda\left(z_{k}\right)=v(k, 0) \in X(\mathcal{T})$. Then $\left|\Delta\left(z_{k}\right)\right|=q^{n_{k}}$ with

$$
\begin{aligned}
n_{k} & =q^{2}+q-q^{1-k} & & k \leqslant 1 \\
& =q^{2}+q+k\left(q^{2}-1\right)-q^{1+k} & & k \geqslant 1 .
\end{aligned}
$$

Proof. Let $z_{0} \in \Omega$ be any element of $\mathbb{F}_{q^{2}}-\mathbb{F}_{q}$. As follows from (1.8), $\lambda\left(z_{0}\right)$ equals the vertex $v(0,0)$. Now $A+A z_{0}=\mathbb{F}_{q^{2}}[T]=: A^{(2)}$, which by the Weierstrass correspondence between lattices and Drinfeld modules corresponds to a rank-one Drinfeld $A^{(2)}$-module $\Phi$. Multiplying $A^{(2)}$ with some constant $\bar{\pi}^{(2)}$ of logarithmic absolute value $\frac{q^{2}}{q^{2}-1}$ (cf. [12]) yields the lattice $\bar{\pi}^{(2)} A^{(2)}$ corresponding to the Carlitz $A^{(2)}$-module $\rho^{(2)}$, defined by the operator polynomial $\rho_{T}^{(2)}(X)=T X+X^{q^{2}}$ (notations as in [7] Section 4). But $\rho^{(2)}$ may be regarded as a rank-two Drinfeld $A$-module with complex multiplication, which yields the discriminant

$$
\begin{aligned}
\Delta\left(z_{0}\right) & =\Delta\left(A^{(2)}\right)=\left(\bar{\pi}^{(2)}\right)^{q^{2}-1} \Delta\left(\bar{\pi}^{(2)} A^{(2)}\right) \\
& =\left(\bar{\pi}^{(2)}\right)^{q^{2}-1} \Delta\left(\rho^{(2)}\right)=\left(\bar{\pi}^{(2)}\right)^{q^{2}-1}
\end{aligned}
$$

of logarithmic absolute value $\log \left|\Delta\left(z_{0}\right)\right|=q^{2}$. Now the formula comes out by inserting (2.9) into (1.13) and integrating.

2.14 Remarks. (i) Since $r(\Delta)$ is linear on edges, we now know $|\Delta(z)|$ for $z \in$ $\lambda^{-1}(A(0, \infty))$. Referring to [8] 6.5, we may determine $|\Delta(z)|$ for arbitrary $z \in \Omega$, provided the coordinates of $\lambda(z)$ on $\mathcal{T}$ (see (1.5)) are specified.

(ii) The infinite product for $\Delta(z)$ given in [4] doesn't suffice to calculate $|\Delta(z)|$ since it converges only for $|z|_{i}$ large, i.e., in the relatively uninteresting case where $\lambda(z)$ is 'close to infinity'.

Next, let $j=\frac{g^{q+1}}{\Delta}$ be the Drinfeld $j$-invariant. It is $\Gamma$-invariant and yields an identification $\Gamma \backslash \Omega \stackrel{\cong}{\longrightarrow} C$. We have $j(z)=0 \Leftrightarrow z \in \Gamma\left(\mathbb{F}_{q^{2}}-\mathbb{F}_{q}\right)$, and all these roots are $q+1$-fold (roots of $g$ are easily verified to be simple: e.g. [7] 5.15). In particular, $j$ is invertible on $\Omega^{\prime}=\Omega-\lambda^{-1}(\Gamma v(0,0))$. Applying the Definition (1.12) of the map $r$ to $j$ yields some function $r(j): Y(\mathcal{T}) \rightarrow \mathbb{Z}$ which is alternating, $\Gamma$-invariant and harmonic (1.6(ii)) at those vertices $v \in X(\mathcal{T})$ which are not $\Gamma$-equivalent to $v(0,0)$.

2.15 CLAIM. At $v \in \Gamma v(0,0)$ we have

$$
\sum_{\substack{e \in Y(\mathcal{T}) \\ o(e)=v}} r(j)(e)=(q+1) q(q-1),
$$


and therefore $r(j)(e)=q(q-1)$ for such $e$, since they are all $\Gamma$-equivalent. It suffices to verify this for $v=v(0,0)$. In $\lambda^{-1}(v)$, we have the $q(q-1)$ zeroes $z \in \mathbb{F}_{q^{2}}-\mathbb{F}_{q}$ of $j$, each with multiplicity $(q+1)$, and no other zeroes. Then the claim follows from the way $r(j)$ has been constructed, i.e., the residue theorem, see [2] and [10] p. 95. Now recall (2.4) that

$$
\Gamma \backslash \mathcal{T}=\stackrel{v_{0}}{e_{0}} \stackrel{e_{0}}{\longrightarrow} \bullet \stackrel{v_{1}}{e_{1}} \stackrel{v_{2}}{\longrightarrow}-\cdots,
$$

where $v_{k}$ is the class of $v(-k, 0), e_{k}$ the class of $e(-k, 0)$. Further, for $k \geqslant 0$, the $q$ positive edges of $\mathcal{T}$ meeting $v(-k-1,0)$ and different from $e(-k-1,0)$ are identified under $\Gamma$ with $e_{k}$. Together, this implies

$$
r(j)\left(e_{k}\right)=q^{k+1}(q-1) .
$$

Let $z_{k} \in \Omega$ be as in (2.13), i.e., $\lambda\left(z_{k}\right)=v(k, 0)$.

2.17 THEOREM. For $0 \neq k \in \mathbb{Z}$ we have

$$
\log \left|j\left(z_{k}\right)\right|=q^{|k|+1} .
$$

Proof. Let $z \in \lambda^{-1}(v(0,0))=\left\{\left.z \in C|| z|=| z\right|_{i}=1\right\}$. All the terms in $E^{(q-1)}(z)=\sum_{a, b}^{\prime} \frac{1}{(a z+b)^{q-1}}$ are $\leqslant 1$ in absolute value, hence $\left\|E^{(q-1)}\right\|_{\lambda^{-1}(v(0,0))} \leqslant$ 1 , and the value 1 is attained e.g. for $z \in \mathbb{F}_{q^{3}}-\mathbb{F}_{q}$. Consequently, $\log \|g\|_{\lambda^{-1}(v(0,0))}=$ $\log \left|T^{q}-T\right|=q$, and from (2.13), $\log \|j\|_{\lambda^{-1}(v(0,0))}=q$. Now for $k<0$,

$$
\log \left|j\left(z_{k}\right)\right|=\int_{v(0,0)}^{v(k, 0)} r(j)(e) \mathrm{d} e+\log \|j\|_{\lambda^{-1}(v(0,0))}
$$

(formula (1.13) is not essentially affected from the defect of harmonicity of $r(j)$ in $v(0,0))$

$$
=q^{-k+1} \text { by }(2.16) \text {. }
$$

For $k>0,\left|j\left(z_{k}\right)\right|=\left|j\left(z_{-k}\right)\right|$ since $v(-k, 0)$ and $v(k, 0)$ are $\Gamma$-equivalent.

2.18 COROLLARY. With the same notation as above,

$$
\begin{aligned}
\log \left|g\left(z_{k}\right)\right| & =q \\
& =q+k(q-1) \quad k \geqslant 1 .
\end{aligned}
$$

Proof. $\log \left|g\left(z_{k}\right)\right|=\frac{1}{q+1}\left(\log \left|\Delta\left(z_{k}\right)\right|+\log \left|j\left(z_{k}\right)\right|\right)$, which yields the result.

2.19 Remark. As for $j, \log |g(z)|$ only depends on $\lambda(z)$ and is linear on edges, as long as $\lambda(z) \notin \Gamma v(0,0)$. The asserted values may be determined directly: The first 
case $\log \left|g\left(z_{k}\right)\right|=q$ for $k \leqslant-1$ reflects that $g=\left(T^{q}-T\right) E^{(q-1)}$ is non-zero at the cusp $\infty$ with $\log |g(\infty)|=q+\log \left|E^{(q-1)}(\infty)\right|=q$, and the second could be seen by inspecting the sum for $E^{(q-1)}\left(z_{k}\right)$. But the crucial point is that $\log |g(z)|$ may be expressed through the corresponding data of $\Delta$ and $j$ even if $\lambda(z) \notin A(0, \infty)$, in which case a direct evaluation of $E^{(q-1)}$ seems difficult.

\section{Roots of modular units}

Let $n \in A$ be monic of degree $\delta>0$, and let $\Gamma_{0}(n)=\left\{\left(\begin{array}{c}a b \\ c d\end{array}\right) \in \Gamma \mid c \equiv 0 \bmod n\right\}$ be the $n$th Hecke congruence subgroup. An elementary calculation yields that $\Delta / \Delta_{n}$ and its $(q-1)$ th root $h / h_{n}$ are modular functions (i.e., invariant) for $\Gamma_{0}(n)$. Correspondingly, $r(\Delta)-r\left(\Delta_{n}\right)$ is a $\Gamma_{0}(n)$-invariant current on $\mathcal{T}$. Here of course $\Delta_{n}(z)=\Delta(n z), h_{n}(z)=h(n z)$. In case $n$ is prime, we determined in [5] Section 4 to what extent roots may be extracted out of $\Delta / \Delta_{n}$ in the function field of the modular curve $X_{0}(n)=\Gamma_{0}(n) \backslash \Omega \cup\{$ cusps $\}$. Here we generalize this result, allowing $n \in A$ arbitrary, and also considering roots in $\mathcal{O}(\Omega)^{*}$.

Let $\varphi \in H(\mathcal{T}, \mathbb{Z})^{\Gamma_{\infty}}$ be given by its Fourier coefficients $c_{0}$ and $c$, and let $\varphi_{n}:=\varphi \circ\left(\begin{array}{l}n \overline{0} \\ 01\end{array}\right) \in \underline{H}(\mathcal{T}, \mathbb{Z})^{\Gamma \infty}$ be its shift by the matrix $\left(\begin{array}{l}n 0 \\ 01\end{array}\right)$.

3.1 LEMMA. $\varphi_{n}$ has the Fourier coefficients $c_{0}^{\prime}, c^{\prime}$ given by

$$
\begin{aligned}
& c_{0}^{\prime}\left(\pi^{k}\right)=q^{\delta} c_{0}\left(\pi^{k}\right) \\
& c^{\prime}\left(\mathfrak{m} \cdot \infty^{k}\right)=c\left(\mathfrak{m} \cdot \operatorname{div}(n)_{f}^{-1} \cdot \infty^{k}\right)
\end{aligned}
$$

( $\mathfrak{m}$ a positive finite divisor).

Proof. The first formula is immediate from (1.16(i)). The second one results from (1.15) and a change of variables.

Next, let $e_{a}, e_{b}, e_{c}$ be the edges $e_{a}=e(2, \pi), e_{b}=e(2,0), e_{c}=e(1,0)$ of $\mathcal{T}$. The picture on $\Gamma_{\infty} \backslash \mathcal{T}$ looks

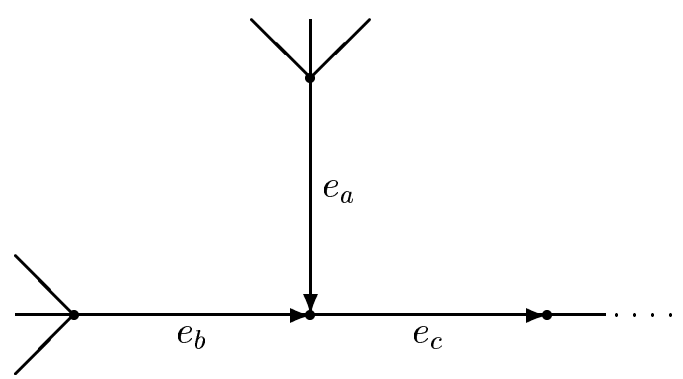


where all the $e(2, t \pi)\left(t \in \mathbb{F}_{q}^{*}\right)$ are identified $\bmod \Gamma_{\infty}$ with $e_{a}$. Thus for $\varphi \in$ $\underline{H}(\mathcal{T}, \mathbb{Z})^{\Gamma \infty}$,

$$
(q-1) \varphi\left(e_{a}\right)+\varphi\left(e_{b}\right)=\varphi\left(e_{c}\right) .
$$

On the other hand, some of the Fourier coefficients of $\varphi$ may directly be evaluated from (1.15):

$$
\begin{aligned}
& c_{0}\left(\pi^{2}\right)=q^{-1}\left((q-1) \varphi\left(e_{a}\right)+\varphi\left(e_{b}\right)\right)=q^{-1} \varphi\left(e_{c}\right), \\
& c((1))=q^{-1}\left(-\varphi\left(e_{a}\right)+\varphi\left(e_{b}\right)\right) .
\end{aligned}
$$

Combining (3.1), (3.3) with (2.6)-(2.9) and solving for the values on the edges $e_{a}, e_{b}, e_{c}$ yields the following table for the functions $\varphi=r(\Delta), r(\Delta)_{n}=r\left(\Delta_{n}\right)$, $r(\Delta)-r\left(\Delta_{n}\right)$.

3.4 TABLE. $(\delta:=\operatorname{deg} n>0)$

\begin{tabular}{llll}
\hline & $r(\Delta)$ & $r\left(\Delta_{n}\right)$ & $r(\Delta)-r\left(\Delta_{n}\right)$ \\
\hline$c((1))$ & $\frac{\left(q^{2}-1\right)(q-1)}{q}$ & 0 & $\frac{\left(q^{2}-1\right)(q-1)}{q}$ \\
$c_{0}(1)$ & $-(q-1) q$ & $-(q-1) q^{\delta+1}$ & $(q-1) q\left(q^{\delta}-1\right)$ \\
$\varphi\left(e_{a}\right)$ & $-(q-1) q$ & $-(q-1) q^{\delta-1}$ & $(q-1) q\left(q^{\delta-2}-1\right)$ \\
$\varphi\left(e_{b}\right)$ & $\left(q^{2}-q-1\right)(q-1)$ & $-(q-1) q^{\delta-1}$ & $(q-1)\left(q^{\delta-1}+q^{2}-q-1\right)$ \\
$\varphi\left(e_{c}\right)$ & $-(q-1)$ & $-(q-1) q^{\delta}$ & $(q-1)\left(q^{\delta}-1\right)$ \\
\hline
\end{tabular}

3.5 COROLLARY. Let $r$ be the largest integer such that there exists an $r$ th root of $\Delta / \Delta_{n}$ in $\mathcal{O}(\Omega)^{*}$. Then $r$ divides $(q-1)^{2}$ if $\delta=\operatorname{deg} n$ is odd, and divides $(q-1)\left(q^{2}-1\right)$ if $\delta$ is even.

(We will see in (3.18) that in fact equality holds.)

Proof. As is immediately verified, $\operatorname{gcd}\left\{\varphi\left(e_{a}\right), \varphi\left(e_{b}\right), \varphi\left(e_{c}\right)\right\}=(q-1)^{2}$, $(q-1)\left(q^{2}-1\right)$ if $\delta$ is odd, even, respectively, for $\varphi=r(\Delta)-r\left(\Delta_{n}\right)$.

In order to construct roots of $\Delta / \Delta_{n}$, we have to introduce some more material.

(3.6) Let $X(n)=\Gamma(n) \backslash \Omega \cup$ \{cusps $\}$ be the Drinfeld modular curve of level $n$, $\Gamma(n)=\left\{\left(\begin{array}{ll}a & b \\ c & d\end{array}\right) \in \Gamma \mid\left(\begin{array}{ll}a & b \\ c & d\end{array}\right) \equiv\left(\begin{array}{ll}1 & 0 \\ 0 & 1\end{array}\right) \bmod n\right\}$. The cusps cusps $(\Gamma(n))$ of $X(n)$ correspond bijectively to

$$
\Gamma(n) \backslash \Gamma / \Gamma_{\infty} \stackrel{\cong}{\longrightarrow} \Gamma(n) \backslash \mathbb{P}^{1}(K) \stackrel{\cong}{\longrightarrow}\left[(A / n)_{\text {prim }}^{2}\right] / \mathbb{F}_{q}^{*},
$$

where $(A / n)_{\text {prim }}^{2} \hookrightarrow(A / n)^{2}$ is the set of pairs $\left\{\begin{array}{l}a \\ c\end{array}\right) \quad \mid a, c \in A / n,(A / n) a+$ $(A / n) c=A / n\}$, and the identification is induced from $\left(\begin{array}{l}a b \\ c d\end{array}\right) \mapsto\left(\begin{array}{l}a \\ c\end{array}\right) \bmod n$. We 
simply write $\left(\begin{array}{l}a \\ c\end{array}\right)$ for the corresponding cusp of $X(n)$. Similarly, the cusps of $X_{0}(n)$ are given by

$$
\operatorname{cusps}\left(\Gamma_{0}(n)\right) \stackrel{\cong}{\longrightarrow} \Gamma_{0}(n) \backslash \Gamma / \Gamma_{\infty} \stackrel{\cong}{\longrightarrow}\left\{\left[\begin{array}{l}
a \\
c
\end{array}\right] \mid a, c \in A / n \text { coprime }\right\},
$$

where $\left[\begin{array}{l}a \\ c\end{array}\right]$ is the equivalence class of $\left(\begin{array}{l}a \\ c\end{array}\right) \bmod \Gamma_{0}(n)$. Let

$$
n=\prod_{1 \leqslant i \leqslant s} f_{i}^{r_{i}}
$$

be the prime decomposition of $n$, i.e., the $f_{i} \in A$ monic, irreducible, of degree $d_{i}$, pairwise different, and put $q_{i}=q^{d_{i}}$. For $x \in A / n$, we let $\underline{h}(x)=\left(h_{1}(x), \ldots, h_{s}(x)\right)$ be its height, where $h_{i}(x)=\operatorname{ord}_{f_{i}}(x) \in\left\{0,1, \ldots, r_{i}\right\}$ is the truncated $f_{i}$-adic valuation. In particular, $h_{i}(0)=r_{i}$. For $\left(\begin{array}{l}a \\ c\end{array}\right) \in \operatorname{cusps}(\Gamma(n))$ we put $\underline{h}\left(\begin{array}{l}a \\ c\end{array}\right)=\underline{h}(c)$ and $\rho\left(\begin{array}{l}a \\ c\end{array}\right)=1$, if there is an $i$ with $0<h_{i}(c)<r_{i}$, and $\rho\left(\begin{array}{l}a \\ c\end{array}\right)=q-1$ otherwise. Note that $\underline{h}\left(\begin{array}{l}a \\ c\end{array}\right)$ and $\rho\left(\begin{array}{l}a \\ c\end{array}\right)$ only depend on the class of $\left(\begin{array}{l}a \\ c\end{array}\right) \bmod \Gamma_{0}(n)$ and therefore are defined on $\operatorname{cusps}\left(\Gamma_{0}(n)\right)$. The next lemma (whose proof we omit) follows from calculating the $\Gamma_{0}(n)$-orbits on $\operatorname{cusps}(\Gamma(n))$.

3.8 LEMMA. The ramification index $\operatorname{ram}\left(\begin{array}{l}a \\ c\end{array}\right)$ of the cusp $\left(\begin{array}{l}a \\ c\end{array}\right)$ of $X(n)$ over the cusp $\left[\begin{array}{l}a \\ c\end{array}\right]$ of $X_{0}(n)$ is given by

$$
\operatorname{ram}\left(\begin{array}{l}
a \\
c
\end{array}\right)=\rho\left(\begin{array}{l}
a \\
c
\end{array}\right) \prod_{1 \leqslant i \leqslant s} q_{i}^{\inf \left\{2 h_{i}, r_{i}\right\}} .
$$

In particular, it depends only on $\underline{h}\left(\begin{array}{l}a \\ c\end{array}\right)=\left(h_{1}, \ldots, h_{s}\right)$.

3.9 EXAMPLE. Let $n$ be prime of degree $\delta$. There are $\left(q^{2 \delta}-1\right) /(q-1) \operatorname{cusps}\left(\begin{array}{l}a \\ c\end{array}\right)$ on $X(n)$ and two cusps $\left[\begin{array}{l}1 \\ 0\end{array}\right]$ and $\left[\begin{array}{l}0 \\ 1\end{array}\right]$ on $X_{0}(n)$. We have $\operatorname{ram}\left(\begin{array}{l}1 \\ 0\end{array}\right)=(q-1) q^{\delta}$ and $\operatorname{ram}\left(\begin{array}{l}0 \\ 1\end{array}\right)=q-1$.

The total ramification index of $\left(\begin{array}{l}a \\ c\end{array}\right)$ over the unique cusp ' $\infty$ ' of $X(1)$ equals $(q-1) \Pi q_{i}^{r_{i}}=(q-1)|n|$, as follows from the description of cusps $(\Gamma(n))$. Since $\Delta$ has a simple zero at $\infty \in X(1)$, we get (with zero orders of modular forms defined as in [6])

$$
\operatorname{ord}_{\left[\begin{array}{c}
a \\
c
\end{array}\right]} \Delta=\frac{(q-1)|n|}{\operatorname{ram}\left(\begin{array}{l}
a \\
c
\end{array}\right)}=\frac{(q-1)}{\rho\left(\begin{array}{l}
a \\
c
\end{array}\right)} \prod_{1 \leqslant i \leqslant s} q_{i}^{r_{i}-\inf \left\{2 h_{i}, r_{i}\right\}}, h_{i}=h_{i}(c) .
$$

Let $w_{n}: z \mapsto \frac{1}{n z}$ be the Atkin-Lehner involution on $\Omega$. The matrix $\left(\begin{array}{l}0 \\ n\end{array}\right)$ normalizes $\Gamma_{0}(n)$ and thus induces an involution on $X_{0}(n)$, which interchanges $\Delta$ and $\Delta_{n}$. 
Furthermore: If $\left[\begin{array}{l}a \\ c\end{array}\right]$ has height $\underline{h}=\left(h_{1}, \ldots, h_{s}\right)$, the cusp $w_{n}\left[\begin{array}{l}a \\ c\end{array}\right]$ has height $\underline{h}^{\prime}=\left(r_{1}-h_{1}, \ldots, r_{s}-h_{s}\right)$. In view of (3.8), we therefore get

$$
\operatorname{ord}_{\left[\begin{array}{c}
a \\
c
\end{array}\right]} \Delta_{n}=\frac{(q-1)|n|}{\operatorname{ram}\left(w_{n}\left(\begin{array}{c}
a \\
c
\end{array}\right)\right)}=\frac{q-1}{\rho\left(\begin{array}{c}
a \\
c
\end{array}\right)} \prod_{1 \leqslant i \leqslant s} q_{i}^{r_{i}-\inf \left\{2\left(r_{i}-h_{i}\right), r_{i}\right\}} .
$$

For any pair $(u, v) \in A \times A-n A \times n A$, we let $e_{u, v}: \Omega \rightarrow C$ be the holomorphic function defined in [3] p. 99, i.e.,

$$
e_{u, v}(z):=e_{\Lambda}\left(\frac{u z+v}{n}\right)
$$

where $\Lambda$ is the $A$-lattice $A z+A \subset C$ and $e_{\Lambda}$ its exponential function. For the moment we are interested in its following properties (loc. cit., in particular Korollar 2.2 and Section 3):

(3.12) (i) $e_{u, v}$ has neither zeroes nor poles on $\Omega$ and depends only on the residue class of $(u, v) \bmod n$.

(ii) $e_{u, v}(\gamma z)=(c z+d)^{-1} e_{(u, v) \gamma}(z), \gamma=\left(\begin{array}{c}a b \\ c d\end{array}\right) \in \Gamma$.

(iii) The inverse $e_{u, v}^{-1}$ is a holomorphic modular form of weight $1, e_{u, v}$ itself is a meromorphic modular form of weight -1 for $\Gamma(n)$.

(iv) The zero order of $e_{u, v}^{-1}$ at $\left(\begin{array}{l}a \\ c\end{array}\right) \in \operatorname{cusps}(\Gamma(n))$ is given by

$$
\operatorname{ord}_{\left(\begin{array}{c}
a \\
c
\end{array}\right)} e_{u, v}^{-1}=|a u+c v|_{n} .
$$

Here $|x|_{n}=\left|x_{0}\right|$ if $x, x_{0} \in A, x \equiv x_{0} \bmod n, \operatorname{deg} x_{0}<\operatorname{deg} n$.

3.13 Remark. The inverse $e_{u, v}^{-1}$ may also be described as the Eisenstein series

$$
e_{u, v}^{-1}(z)=E_{u, v}(z)=\sum_{\substack{a, b \in A \\(a, b) \equiv(u, v) \bmod n}}^{\prime}(a z+b)^{-1} .
$$

This will however not be used in this paper.

We now define the functions on $\Omega$

$$
\begin{aligned}
& F(z)=\prod_{\substack{0 \neq v \in A \\
\operatorname{deg} v<\delta}} e_{0, v}^{-1}, \\
& G(z)=\prod_{\substack{v \text { monic } \\
\operatorname{deg} v<\delta}} e_{0, v}^{-1} .
\end{aligned}
$$

Then clearly $F=(-1)^{\delta} G^{q-1}$, and $F$ is modular of weight $|n|-1$ and type 0 for $\Gamma_{0}(n)$, as immediately results from the transformation rule of the $e_{u, v}$. Further, its orders at the various cusps of $X_{0}(n)$ are

$$
\operatorname{ord}_{\left[\begin{array}{c}
a \\
c
\end{array}\right]} F=\operatorname{ram}\left(\begin{array}{c}
a \\
c
\end{array}\right)^{-1} \sum_{0 \neq v \in A / n}|v c|_{n} .
$$


3.16 THEOREM. The divisors of $\Delta, \Delta_{n}$ and $F$ on $X_{0}(n)$ are related by

$|n| \operatorname{div} \Delta-\operatorname{div} \Delta_{n}=\left(q^{2}-1\right) \operatorname{div} F$.

Proof. All the divisors have their support in $\operatorname{cusps}\left(\Gamma_{0}(n)\right)$, so we have to compare their orders at the different cusps. Those of $\Delta$ and $\Delta_{n}$ are given by (3.8), (3.10) and (3.11). They only depend on the height $\underline{h}=\left(h_{1}, \ldots, h_{s}\right)$ of the cusp $\left[\begin{array}{c}a \\ c\end{array}\right]$, as is the case for ord ${ }_{\left[\begin{array}{c}a \\ c\end{array}\right]} F$. Thus we may without restriction assume that $c$ is a divisor of $n, c=\prod_{1 \leqslant i \leqslant s} f_{i}^{h_{i}}$. For such $c$, we find by an elementary calculation

$$
\sum_{0 \neq v \in A / n}|v c|_{n}=\frac{|n|^{2}-|c|^{2}}{q+1},
$$

where $|c|=\prod_{1 \leqslant i \leqslant s} q_{i}^{h_{i}}$. Hence for an arbitrary cusp $\left[\begin{array}{c}a \\ c\end{array}\right]$ of height $\underline{h}$,

$$
\sum_{0 \neq v \in A / n}|v c|_{n}=\frac{|n|^{2}-\Pi q_{i}^{h_{i}}}{q+1} .
$$

Inserting this into (3.15) yields the result.

3.17 COROLLARY. Up to constants we have

$$
\frac{\Delta}{\Delta_{n}}=\text { const. } \frac{F^{q^{2}-1}}{\Delta^{|n|-1}}
$$

3.18 COROLLARY. The estimate given in (3.5) for the root number $r$ is sharp. that is, $\Delta / \Delta_{n}$ has an rth root in $\mathcal{O}(\Omega)^{*}$, where $r=(q-1)^{2}$ if $\delta$ is odd and $r=(q-1)\left(q^{2}-1\right)$ if $\delta$ is even, and $r$ is maximal.

Proof. Recall first that $\Delta=-h^{q-1}$ and $F=$ const. $G^{q-1}$ are $(q-1)$ th powers. Thus $r \geqslant(q-1) \operatorname{gcd}\left\{\left(q^{2}-1\right),|n|-1\right\}$, which is as stated.

The function $G$ is a modular form of weight $(|n|-1) /(q-1)$ for $\Gamma_{1}(n)=\left\{\begin{array}{l}a b \\ c d\end{array}\right) \in$ $\Gamma \mid a \equiv d \equiv 1, c \equiv 0 \bmod n\}$ and transforms according to

$$
G(\gamma z)=\chi(\gamma)(c z+d)^{(|n|-1) /(q-1)} G(z)
$$

under $\Gamma_{0}(n)$, where $\chi$ is a character with $\chi^{q-1}=1$, i.e., $\chi: \Gamma_{0}(n) \rightarrow \mathbb{F}_{q}^{*}$. We will next determine $\chi$. Recall that $n=\Pi f_{i}^{r_{i}}$ as usual. For $1 \leqslant i \leqslant s$, we let

$$
N_{i}:(A / n)^{*} \rightarrow\left(A / f_{i}\right)^{*} \rightarrow \mathbb{F}_{q}^{*}
$$

be the canonical projection followed by the norm. 
3.20 THEOREM. The 'nebentype' $\chi$ of $G$ is given by

$$
\begin{aligned}
\chi: \Gamma_{0}(n) & \rightarrow \mathbb{F}_{q}^{*} \\
\left(\begin{array}{l}
a b \\
c d
\end{array}\right) & \mapsto \Pi N_{i}(d)^{-r_{i}} .
\end{aligned}
$$

Proof. First note that for $a \in \mathbb{F}_{q}^{*}$

$$
e_{0, a v}(z)=a \cdot e_{0, v}(z) .
$$

Let $S \subset A / n-\{0\}$ be the set of monics, which is a set of representatives for $(A / n-\{0\}) / \mathbb{F}_{q}^{*}$, as is $\mathrm{d} S$ if $d \in(A / n)^{*}$. Hence for each $v \in S$ there are unique $a_{v} \in \mathbb{F}_{q}^{*}, v^{\prime} \in S$ such that $d v=a_{v} \cdot v^{\prime}, d$ being fixed. In view of (1) and the definition of $G$, we will have

$$
\chi\left(\left(\begin{array}{ll}
a & b \\
c & d
\end{array}\right)\right)=\prod_{v \in S} a_{v}^{-1} .
$$

For each height vector $\underline{h}=\left(h_{1}, \ldots, h_{s}\right)<\underline{r}=\left(r_{1}, \ldots, r_{s}\right)$, we let $(A / n)(\underline{h})$ be the elements of $A / n$ of height $\underline{h}$ and $S(\underline{h})$ the monics in $(A / n)(\underline{h})$. We calculate the contribution of $S(\underline{h})$ to (2).

Let $m=m(\underline{h}):=\Pi f_{i}^{r_{i}-h_{i}}$. Then the group $(A / m)^{*}$ acts faithfully and simply transitively on $(A / n)(\underline{h})$, that is, for $v, v^{\prime} \in(A / n)(\underline{h})$ there exists a unique $x \in$ $(A / m)^{*}$ such that $x v=v^{\prime}$, labelled $\left(\frac{v^{\prime}}{v}\right)$. Then

$$
\prod_{v \in S(\underline{h})} a_{v}=\prod_{v \in S(\underline{h})}\left(\frac{d v}{v}\right)=(d \bmod m)^{\varphi(m) /(q-1)},
$$

since $d S(\underline{h})$ and $S(\underline{h})$ are representatives for $(A / n)(\underline{h}) / \mathbb{F}_{q}^{*}$. Here $\varphi(m):=\sharp(A / m)^{*}$, and the right hand term lies in $\mathbb{F}_{q}^{*} \hookrightarrow(A / m)^{*}$. Now

$$
(A / m)^{*} \stackrel{\cong}{\longrightarrow} \prod_{i \text { s.t. } h_{i}<r_{i}}\left(A / f_{i}^{r_{i}-h_{i}}\right)^{*}
$$

which implies $(d \bmod m)^{\varphi(m) /(q-1)}=1$ if $m$ fails to be primary, i.e., if there are at least two $i$ with $h_{i}<r_{i}$. If $m=f_{i}^{r_{i}-h_{i}}$ is primary, $(A / m)^{*}$ decomposes canonically into $\left(A / f_{i}\right)^{*}$ and its $p$-Sylow group of order $q_{i}^{r_{i}-h_{i}-1}$, and $(d \bmod m)^{\varphi(m) /(q-1)}=$ $\left(d \bmod f_{i}\right)^{\left(q_{i}-1\right) /(q-1)}=N_{i}(d)$. Hence

$$
\begin{aligned}
\prod_{v \in S(\underline{h})} a_{v} & =N_{i}(d), & & \exists ! i \text { s.t. } h_{i}<r_{i} \\
& =1 & & \text { otherwise. }
\end{aligned}
$$


Inserting (4) into (2) finishes the proof.

For $\delta=\operatorname{deg} n$ even (odd), let $D_{n}$ be the function $G \cdot h^{-(|n|-1) /\left(q^{2}-1\right)}$ $\left(G^{q+1} \cdot h^{-(|n|-1) /(q-1)}\right)$, respectively, i.e., $\Delta / \Delta_{n}=$ const. $D_{n}^{r}$ with $r$ as in (3.18).

3.21 COROLLARY. The function $D_{n}$ transforms under $\Gamma_{0}(n)$ according to the character $\omega_{n}:=\chi \cdot \operatorname{det}^{\delta / 2}$ if $\delta$ is even and $\omega_{n}=\chi^{2} \cdot \operatorname{det}^{\delta}$ if $\delta$ is odd.

Proof. For $\delta$ odd,

$$
\begin{aligned}
D_{n}(\gamma z)= & \frac{\chi^{q+1}(\gamma)}{\operatorname{det}(\gamma)^{-(|n|-1) /(q-1)}} D_{n}(z) \\
& \left(\text { by the theorem and the definition of } D_{n}\right) \\
= & \chi^{2}(\gamma) \operatorname{det}(\gamma)^{\delta} D_{n}(z),
\end{aligned}
$$

since $\frac{|n|-1}{q-1}=\frac{q^{\delta}-1}{q-1} \equiv \delta \bmod q-1$. A similar consideration gives the result for $\delta$ even.

Let $o\left(\omega_{n}\right)$ be the order of $\omega_{n}$. Then $D_{n}^{o\left(\omega_{n}\right)}$ is the least power of $D_{n}$ which is $\Gamma_{0}(n)$-invariant, and $r / o\left(\omega_{n}\right)$ is the largest number $k$ such that $\Delta / \Delta_{n}$ has a $k$ th root in the field of modular functions for $\Gamma_{0}(n)$.

\subsection{PROPOSITION.}

$$
\begin{aligned}
o\left(\omega_{n}\right) & =\frac{q-1}{\operatorname{gcd}\left(q-1, r_{1}, \ldots, r_{s}, \delta / 2\right)}, \delta \text { even } \\
& =\frac{q-1}{\operatorname{gcd}\left(q-1, r_{1}, \ldots, r_{s}, \delta\right)}, \quad \delta \text { odd } .
\end{aligned}
$$

Proof. For any of the characters $N_{i}^{-r_{i}}$ (see (3.20)), $\chi$, det, $\omega_{n}$, its order is the size of its image in $\mathbb{F}_{q}^{*}$. E.g. for $N_{i}^{-r_{i}}$, it equals $\frac{q-1}{\operatorname{gcd}\left(q-1, r_{i}\right)}$ since $N_{i}: \Gamma_{0}(n) \rightarrow \mathbb{F}_{q}^{*}$, $\left(\begin{array}{l}a b \\ c d\end{array}\right) \mapsto N_{i}(d)$, is surjective. The assertion now follows from the Chinese remainder theorem.

In the concluding corollaries, we let ' 0 ' $=\left[\begin{array}{l}0 \\ 1\end{array}\right]$ ' $\infty$ ' $=\left[\begin{array}{l}1 \\ 0\end{array}\right]$ be the distinguished cusps of $X_{0}(n)$.

3.23 COROLLARY (see [5]). Let $n$ be prime of degree $\delta$. The cuspidal divisor class group $\mathcal{C}$ of $X_{0}(n)$ is cyclic of order $(|n|-1) /\left(q^{2}-1\right)$ if $\delta$ is even and $(|n|-1) /(q-1)$ if $\delta$ is odd.

Proof. By (3.9), $\mathcal{C}$ is the group generated by the class of $[(0)-(\infty)]$. The divisor of $\Delta / \Delta_{n}$ is $(|n|-1)[(0)-(\infty)]$, and the character $\omega_{n}$ of $D_{n}$ has exact order 
$(q-1)$. Hence $D_{n}^{q-1}$ but no smaller power of $D_{n}$ is invariant under $\Gamma_{0}(n)$, and the class of $[(0)-(\infty)]$ has the asserted order.

3.24 Remark. In the above situation, let $t$ be the gcd of $q-1$ and $\sharp \mathcal{C}$. Then yet the divisor of $D_{n}^{(q-1) / t}$ on $X(n)$ comes from a divisor on $X_{0}(n)$, and $(q-1) / t$ is minimal with that property. As we will show in subsequent work, this implies that the kernel of the canonical map from $\mathcal{C}$ to the group $\Phi_{\infty}$ of connected components of the Nerron model $/ K_{\infty}$ of the Jacobian $\operatorname{Jac}\left(X_{0}(n)\right)$ is the subgroup of order $t$ in $\mathcal{C}$. Hence the picture differs significantly from the one at the finite place $(n)$, where the corresponding mapping $\mathcal{C} \rightarrow \Phi_{(n)}$ is always bijective [5]. This gives a negative answer to a question raised by J. Teitelbaum ([18] p. 283).

3.25 COROLLARY. Let $n=f^{2}$, $f$ prime. The divisor class of $[(0)-(\infty)]$ in $X_{0}(n)$ has order $(|n|-1) /\left(q^{2}-1\right)$ is $q$ is even or $\operatorname{deg} f$ is odd, and $(|n|-1) / 2\left(q^{2}-1\right)$ if $q$ is odd and deg $f$ is even.

Proof. Besides 0 and $\infty$, there are $(|f|-1) /(q-1)$ cusps $s=\left[\begin{array}{l}u \\ f\end{array}\right]$ of height 1 ( $u$ monic of degree $<\operatorname{deg} f$ ). Now $\operatorname{ord}_{s} \Delta=\operatorname{ord}_{s}\left(\Delta_{n}\right)=q-1$ for such $s$, and thus $\operatorname{div}\left(\Delta / \Delta_{n}\right)=(|n|-1)[(0)-(\infty)]$. We conclude with (3.22).

We believe that an extension of the preceding arguments eventually will lead to the determination of the cuspidal divisor class groups $\mathcal{C}$ of all the curves $X_{0}(n)$, $X_{1}(n), X(n)$, where $n$ is a not necessarily prime element of $A$. A first step has been carried out in [3], from whose results upper estimates for $\sharp \mathcal{C}$ may be derived.

\section{References}

1. Deligne, P., Husemöller, D.: Survey of Drinfeld modules, Contemp. Math. 67 (1987) 25-91.

2. Fresnel, J., van der Put, M.: Géométrie analytique rigide et applications, Progr. Math. 18 (1981), Birkhäuser, Basel, Boston.

3. Gekeler, E.-U.: Modulare Einheiten für Funktionenkörper. J. reine angew. Math. 348 (1984), 94-115.

4. Gekeler, E.-U.: A product expansion for the discriminant function of Drinfeld modules of rank two. J. Number Theory 21 (1985), 135-140.

5. Gekeler, E.-U.: Über Drinfeld'sche Modulkurven vom Hecke-Typ. Comp. Math. 57 (1986), 219-236.

6. Gekeler, E.-U.: Drinfeld Modular Curves, Lect. Notes Math., vol. 1231, Springer, Berlin, Heidelberg, New York, 1986.

7. Gekeler, E.-U.: On the coefficients of Drinfeld modular forms, Invent. Math. 93 (1988), 667-700.

8. Gekeler, E.-U.: Improper Eisenstein series on Bruhat-Tits trees, manuscripta math. 86 (1995), 367-391.

9. Gekeler, E.-U., Reversat, M.: Jacobians of Drinfeld modular curves. J. reine angew. Math. 476 (1996), 27-93.

10. Gerritzen, L., van der Put, M.: Schottky Groups and Mumford Curves. Lect. Notes Math., vol. 817, Springer, Berlin, Heidelberg, New York, 1980.

11. Goldman, O., Iwahori, N.: The space of $p$-adic norms. Acta Math. 109 (1963), 137-177.

12. Goss, D.: Von Staudt for $\mathbb{F}_{q}[T]$, Duke Math. J. 45 (1978), 885-910.

13. Goss, D.: $\pi$-adic Eisenstein Series for Function Fields, Comp. Math. 41 (1980), 3-38.

14. Goss, D.: The algebraist's upper half-plane. Bull. AMS NS 2 (1980), 391-415. 
15. Robert, G.: Concernant la relation de distribution satisfaite par la fonction $\varphi$ associée à un réseau complexe. Invent. Math. 100 (1990), 231-257.

16. Robert, G.: La racine 12-ième canonique de $\Delta(L)^{[\underline{L}: L]} / \Delta(\underline{L})$. In: Séminaire de Théorie des Nombres 1989/90, S. David (ed.), Progr. Math. 102 (1992), Birkhäuser, Basel, Boston.

17. Serre, J.-P.: Trees. Springer, Berlin, Heidelberg, New York, 1980.

18. Teitelbaum, J.: Modular symbols for $\mathbb{F}_{q}(T)$. Duke Math. J. 68 (1992), 271-295.

19. Weil, A.: Dirichlet series and automorphic forms, Lect. Notes Math., vol. 189, Springer, Berlin, Heidelberg, New York, 1971. 